(1)

CrossMark

\title{
Risk assessment in pulmonary arterial hypertension
}

\author{
To the Editor:
}

In its August 2017 issue, the European Respiratory Journal published two papers on risk assessment in pulmonary arterial hypertension $(\mathrm{PAH})$, one coming from the French registry [1], the other from COMPERA, a European pulmonary hypertension $(\mathrm{PH})$ registry [2]. Both groups utilised abbreviated versions of the risk assessment strategy proposed in the current European PH guidelines [3, 4] and both included basically the same variables, i.e. World Health Organization functional class (FC), 6-min walking distance (6MWD), cardiac index, right atrial pressure and serum levels of brain natriuretic peptide (BNP) or the N-terminal fragment of its propeptide (NT-proBNP). In both studies, the cut-off values used to determine risk were those proposed in the European guidelines [3, 4]. However, the strategy to determine individual risk differed in the two studies: COMPERA calculated the average individual risk by assigning a score of 1,2 or 3 to each criterion (1: low risk; 2; intermediate risk; 3 : high risk) and rounding to the mean of the available variables, as had been previously proposed by a group from Sweden [5]. The French group, in contrast, looked at the number of variables that met the low-risk criteria. Despite these differences, the main results of both studies were similar, especially 1) that the risk stratification tool proposed by the European guidelines adequately predicted mortality, 2) that follow-up risk assessment on treatment was a more reliable predictor of survival than the initial risk assessment at the time of diagnosis, and 3) that FC, 6MWD and BNP/NT-proBNP at follow-up were independent predictors of outcome. However, there also were important differences in the two analyses: in the French study, 19\% of the patients with idiopathic PAH (IPAH) met all three noninvasive low-risk criteria at follow-up, i.e. FC I or II, $6 \mathrm{MWD}>440 \mathrm{~m}, \mathrm{BNP}<50 \mathrm{ng} \cdot \mathrm{L}^{-1}$ or NT-proBNP $<300 \mathrm{ng} \cdot \mathrm{L}^{-1}$; these patients had a 1-year survival of $100 \%$ and a 5 -year survival of $97 \%$. In COMPERA, $21 \%$ of the IPAH patients were classified as low risk at follow-up; these patients had a 1-year survival of $96 \%$ but the 5 -year survival of these patients was only $72 \%$ (data available in the online supplement of [2]). We wondered whether the differences in long-term survival were due to the fact that the low-risk definition was less strict with the COMPERA strategy than with the French strategy or whether differences in the two patient cohorts were responsible for these findings (patients in French cohort were younger than the patients in COMPERA and combinations of PAH drugs were used more frequently in the French series than in the COMPERA cohort).

In order to address these questions, we revisited the COMPERA cohort that was analysed for the recent risk assessment paper [2] and applied the French risk assessment strategy [1] to 579 IPAH patients for whom complete data sets of FC, 6MWD and BNP/NT-proBNP were available at first follow-up (median 4.6 months, interquartile range 3.1-9.5 months) after treatment initiation. This cohort represented $77 \%$ of the 756 IPAH patients from the original manuscript for whom follow-up data were available. The baseline characteristics and treatment patterns of this subgroup were identical to the entire cohort [2]. The three dichotomised low-risk criteria remained independently associated with survival in a multivariate Cox model adjusted for age and sex. The hazard ratios (95\% CI) were as follows. FC I/II: $0.48(0.30-0.75)$, $\mathrm{p}=0.001 ; 6 \mathrm{MWD}>440 \mathrm{~m}: 0.34(0.16-0.69), \mathrm{p}=0.003 ; \mathrm{NT}-\mathrm{proBNP}<300 \mathrm{ng} \cdot \mathrm{L}^{-1}$ or $\mathrm{BNP}<50 \mathrm{ng} \cdot \mathrm{L}^{-1}: 0.54$ (0.33-0.88), $\mathrm{p}=0.013$.

When the French noninvasive risk assessment strategy was applied to the COMPERA cohort, the estimated survival rates at 1,3 and 5 years of patients meeting all three low-risk criteria at follow-up were $100 \%, 100 \%$ and $95 \%$, respectively. The corresponding survival rates were $99 \%, 95 \%$ and $76 \%$ for patients meeting two low-risk criteria; $93 \%, 75 \%$ and $64 \%$ for patients meeting one low-risk criterion; and $90 \%$, $61 \%$ and $43 \%$ for patients who had no low-risk criteria at follow-up ( $<<0.001$ by log-rank test; figure 1$)$. These figures were comparable with the corresponding survival rates observed in the French cohort [1].

@ERSpublications

PAH patients reaching a low risk profile with targeted therapies have an excellent long-term survival http://ow.ly/RBjo30i6N57

Cite this article as: Hoeper MM, Pittrow D, Opitz C, et al. Risk assessment in pulmonary arterial hypertension. Eur Respir J 2018; 51: 1702606 [https://doi.org/10.1183/13993003.02606-2017]. 
FIGURE 1 Kaplan-Meier survival estimates according to the number of low-risk criteria met at first follow-up (see main text for details). $p<0.001$.

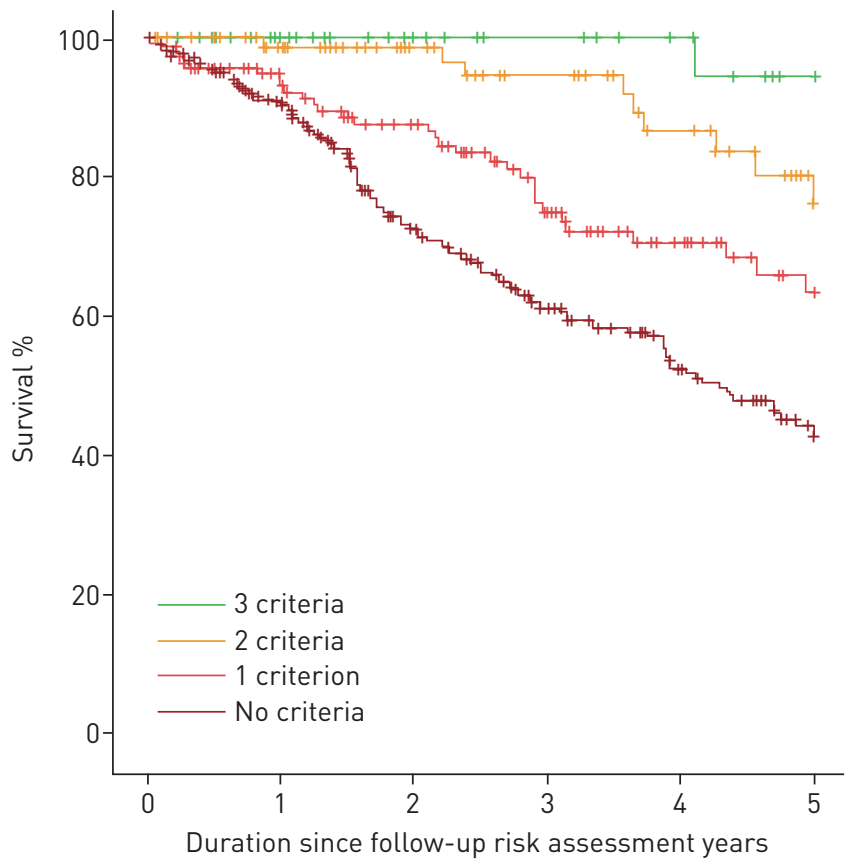

In the present analysis, at baseline, three, two, one and no low-risk criteria were present in $3 \%, 8 \%, 21 \%$ and $69 \%$ of the patients, respectively. From baseline to first follow-up, 5\% of the patients worsened, $64 \%$ remained unchanged and $31 \%$ improved their number of low-risk criteria. At first follow-up, three, two, one and no low-risk criteria were found in $9 \%, 15 \%, 24 \%$ and $52 \%$ of the patients, respectively. The corresponding numbers in the French cohort were 19\%, 24\%, 29\% and 28\%, respectively. In other words, only $48 \%$ of the COMPERA patients reached at least one noninvasive low-risk criterion at first follow-up compared to $72 \%$ of the patients in the French registry. Differences in treatment strategies may have contributed to the better long-term survival in the French cohort, where early combinations of PAH drugs were used more frequently than in the COMPERA cohort $[1,2]$. However, it is likely that the age differences between the two groups contributed to the survival differences as well. The average age at inclusion was $64 \pm 16$ years in COMPERA versus $57 \pm 17$ years in the French cohort. In the present analysis, there was a tight relationship between the likelihood of reaching low-risk criteria and age at time of diagnosis: the mean $\pm \mathrm{SD}$ age of patients meeting all three noninvasive low-risk criteria at follow-up was $45 \pm 18$ years; the respective age of patients meeting two, one or no low-risk criteria was $57 \pm 16,65 \pm 15$ and $68 \pm 14$ years. Still, the observed survival differences between the risk strata remained statistically significant $(\mathrm{p}<0.001$ in Cox regression analysis) when adjusted for age and sex.

In summary, the present data support the notion that the French risk assessment strategy provides a more accurate identification of patients with an excellent long-term survival than the approach of averaging risk scores as originally applied by the Swedish group [5] and the COMPERA investigators [2]. Patients meeting low-risk criteria for FC, 6MWD and BNP/NT-proBNP at first follow-up on therapy had a 5-year survival rate of $97 \%$ in the French registry and of $95 \%$ in COMPERA. However, only 19\% of the French patients and $9 \%$ of the COMPERA patients met these criteria at first follow-up. Our results indicate that the likelihood of reaching a low-risk profile declines with increasing age, but it is unclear how much this is attributable to changes in PAH phenotypes, comorbidities or a tendency to use less aggressive PAH treatment strategies in elderly patients with IPAH [6].

Marius M. Hoeper ${ }^{1,2}$, David Pittrow ${ }^{3}$, Christian Opitz ${ }^{4}$, J. Simon R. Gibbs ${ }^{5}$, Stephan Rosenkranz ${ }^{6,7}$, Ekkehard Grünig ${ }^{2,8}$, Karen M. Olsson ${ }^{1,2}$ and Doerte Huscher ${ }^{9}$

${ }^{1}$ Dept of Respiratory Medicine, Hannover Medical School, Hannover, Germany. ${ }^{2}$ German Center of Lung Research (DZL), Germany. ${ }^{3}$ Institute for Clinical Pharmacology, Medical Faculty, Technical University, Dresden, Germany. ${ }^{4}$ Dept of Cardiology, DRK Kliniken Berlin Westend, Berlin, Germany. ${ }^{5}$ Dept of Cardiology, National Heart and Lung Institute, Imperial College London, London, UK. ${ }^{6}$ Clinic III for Internal Medicine (Cardiology) and Center for Molecular Medicine (CMMC), University of Cologne, Cologne, Germany. ${ }^{7}$ The Cologne Cardiovascular Research Center (CCRC), University of Cologne, Cologne, Germany. ${ }^{8}$ Thoraxclinic at the University Hospital Heidelberg, Heidelberg, Germany. ${ }^{9}$ Epidemiology Unit, German Rheumatism Research Centre, A. Leibniz Institute, Berlin, Germany.

Correspondence: Marius M. Hoeper, Dept of Respiratory Medicine, Hannover Medical School, 30623 Hannover, Germany. E-mail: hoeper.marius@mh-hannover.de 
Received: Dec 152017 | Accepted after revision: Jan 022018

Acknowledgements: The authors are indebted to the COMPERA investigators.

Conflict of interest: M.M. Hoeper reports receiving personal fees from Actelion, Bayer, GSK, Pfizer, Gilead and Merck, during the conduct of the study. D. Pittrow reports receiving personal fees from Actelion, Aspen, Bayer, Boehringer Ingelheim, Daiichi Sankyo, Novartis, Pfizer and AstraZeneca, outside the submitted work. C. Opitz reports receving personal fees from Actelion, Bayer, GSK, Pfizer and Novartis, during the conduct of the study. J.S.R. Gibbs reports receiving personal fees from Actelion, Bayer, GSK, Pfizer, MSD, Bellerophon and Arena, during the conduct of the study. S. Rosenkranz reports receving grants and personal fees from Actelion, Bayer, Pfizer, Novartis and United Therapeutics, and personal fees from GSK, Gilead and MSD, during the conduct of the study. E. Grünig reports receiving personal fees from Actelion, Bayer, GSK, Pfizer, United Therapeutics and MSD, outside the submitted work. K.M. Olsson reports receiving personal fees from Actelion, Bayer, GSK, Pfizer and United Therapeutics, during the conduct of the study. D. Huscher reports receiving personal fees from Actelion outside the submitted work.

Support statement: This work was supported by the DZL and the Deutsche Forschungsgemeinschaft (to M.M. Hoeper, grant number HO 1599/2-1). COMPERA is funded by unrestricted grants from Actelion Pharmaceuticals, Bayer and GSK. These companies were not involved in data analysis or the writing of this manuscript. Funding information for this article has been deposited with the Crossref Funder Registry.

\section{References}

1 Boucly A, Weatherald J, Savale L, et al. Risk assessment, prognosis and guideline implementation in pulmonary arterial hypertension. Eur Respir J 2017; 50: 1700889.

2 Hoeper MM, Kramer T, Pan Z, et al. Mortality in pulmonary arterial hypertension: prediction by the 2015 European pulmonary hypertension guidelines risk stratification model. Eur Respir J 2017; 50: 1700740.

3 Galie N, Humbert M, Vachiery JL, et al. 2015 ESC/ERS Guidelines for the diagnosis and treatment of pulmonary hypertension. Eur Respir J 2015; 46: 903-975.

4 Galie N, Humbert M, Vachiery JL, et al. 2015 ESC/ERS Guidelines for the diagnosis and treatment of pulmonary hypertension. Eur Heart J 2016; 37: 67-119.

5 Kylhammar D, Kjellstrom B, Hjalmarsson C, et al. A comprehensive risk stratification at early follow-up determines prognosis in pulmonary arterial hypertension. Eur Heart J 2017 in press [https://doi.org/10.1093/eurheartj/ehx257].

6 Opitz CF, Hoeper MM, Gibbs JS, et al. Pre-capillary, combined, and post-capillary pulmonary hypertension: a pathophysiological continuum. J Am Coll Cardiol 2016; 68: 368-378.

Copyright @ERS 2018

From the authors:

We read with interest the correspondence by M.M. Hoeper and colleagues regarding risk assessment in pulmonary arterial hypertension (PAH). This follows the three manuscripts on the topic published in 2017 in the European Respiratory Journal [1,2] and in the European Heart Journal [3]. All utilised a risk-assessment method derived from the risk-stratification table proposed by the 2015 European Society of Cardiology/European Respiratory Society Guidelines for the diagnosis and treatment of pulmonary hypertension $[4,5]$.

Two different methods were utilised to assess risk in large cohorts of patients with PAH. In their respective registries, Kylhammar et al. [3] and Hoeper et al. [2] calculated an averaged risk by assigning a score of 1,2 or 3 for each criterion according to the level of risk ( 1 for a low-risk, 2 for an intermediate-risk and 3 for a high-risk criterion). Then, the sum of the score obtained was divided by the number of available variables for each patient and was rounded off to the nearest integer, defining an individual risk score corresponding to the patient's risk group $(1,2$ or 3 , for low, intermediate or high risk, respectively) [2, 3]. In the French registry, our approach consisted of counting the number of low-risk criteria present at baseline and then achieved at first follow-up [1]. Two models were analysed in our study. In the first model, four variables were taken into account: New York Heart Association (NYHA)/ World Health Organization (WHO) functional class, 6-min walking distance (6MWD), right atrial pressure and cardiac index measured by right-heart catheterisation. The second model was one using only

@ERSpublications

Risk stratification of pulmonary arterial hypertension based on the number of noninvasive low-risk criteria achieved at first follow-up provides an accurate identification of patients with an excellent prognosis http://ow.ly/IyNI30irqpK

Cite this article as: Boucly A, Weatherald J, Humbert $\mathrm{M}$, et al. Risk assessment in pulmonary arterial hypertension. Eur Respir J 2018; 51: 1800279 [https://doi.org/10.1183/13993003.00279-2018]. 
the presence or the achievement of three noninvasive low-risk criteria: NYHA/WHO functional class I-II, $6 \mathrm{MWD}>440 \mathrm{~m}$ and $\mathrm{N}$-terminal pro-brain natriuretic peptide (NT-proBNP) $<300 \mathrm{ng} \cdot \mathrm{L}^{-1}$ or brain natriuretic peptide $(\mathrm{BNP})<50 \mathrm{ng} \cdot \mathrm{L}^{-1}$. In both models, with increasing number of low-risk criteria achieved at first follow-up evaluation, transplant-free survival was better [1].

In their correspondence, M.M. Hoeper and colleagues also utilised the French registry methodology [1] for the risk assessment of patients with PAH enrolled in the COMPERA registry. This study represents an external validation of the use of three simple noninvasive low-risk criteria to identify patients with idiopathic PAH having an excellent long-term prognosis (5-year survival rate of 95\%).

We recently performed additional analyses in our cohort to determine the validity of our two models. We determined the index of concordance by Harrell's c-index in the model with four variables (including invasive haemodynamic parameters) and in the model that included three noninvasive variables [6]. Values for the c-index were higher than 0.7 for the models based on variables at first follow-up, indicating that our two models are strong: Harrell's c-index of 0.72 for the invasive model and 0.74 for the noninvasive one. However, for the multivariable analysis based on variables present at baseline, concordance was less strong, with a Harrell's c-index of 0.61 for the invasive model. These results confirm that low-risk criteria achieved at first follow-up were more discriminant for predicting survival than those present at baseline.

To conclude, our study and the analyses performed in the COMPERA registry confirm that our approach of risk stratification based on the number of noninvasive low-risk criteria achieved at first follow-up provides an accurate identification of patients with an excellent prognosis. The achievement of the three noninvasive low-risk criteria (NYHA/WHO class I-II, 6MWD $>440 \mathrm{~m}$ and NT-proBNP $<300 \mathrm{ng} \cdot \mathrm{L}^{-1}$ or BNP $<50 \mathrm{ng} \cdot \mathrm{L}^{-1}$ ) could be considered as a treatment goal in patients with PAH. However, only few patients reached those three low-risk criteria at first follow-up (9\% in the COMPERA cohort and 19\% in the French cohort). Those results suggest that there is still more to be done to obtain a greater proportion of patients reaching all low-risk criteria.

Athénaïs Boucly ${ }^{1,2,3}$, Jason Weatherald $\oplus^{4}$, Marc Humbert $\oplus^{1,2,3}$ and Olivier Sitbon $\oplus^{1,2,3}$

${ }^{1}$ Université Paris-Sud, Faculté de Médecine, Université Paris-Saclay, Le Kremlin-Bicêtre, France. ${ }^{2}$ AP-HP, Service de Pneumologie, Hôpital Bicêtre, Le Kremlin-Bicêtre, France. ${ }^{3}$ INSERM UMR_S 999, Hôpital Marie Lannelongue, Le Plessis Robinson, France. ${ }^{4}$ University of Calgary, Dept of Medicine, Division of Respirology, Calgary, AB, Canada.

Correspondence: Olivier Sitbon, Service de Pneumologie, Hôpital Bicêtre, 78 avenue du Général Leclerc, 94275 Le Kremlin-Bicêtre, France. E-mail: olivier.sitbon@bct.aphp.fr

Received: Feb 062018 | Accepted after revision: Feb 072018

Conflict of interest: A. Boucly reports personal fees and non-financial support from Actelion, and non-financial support from GlaxoSmithKline and Merck, outside the submitted work. J. Weatherald reports grants from the European Respiratory Society and the Canadian Thoracic Society, during the conduct of the study, as well as personal fees and non-financial support from Actelion Pharmaceuticals and non-financial support from Bayer, for travel to scientific meetings outside the submitted work. M. Humbert reports grants, personal fees and non-financial support from Actelion, Bayer, GlaxoSmithKline and Merck, and personal fees from Arena and Novartis, all outside the submitted work. O. Sitbon reports grants, personal fees and non-financial support from Actelion, Bayer, GlaxoSmithKline and Merck, and personal fees from Arena, all outside the submitted work.

\section{References}

1 Boucly A, Weatherald J, Savale L, et al. Risk assessment, prognosis and guideline implementation in pulmonary arterial hypertension. Eur Respir J 2017; 50: 1700889.

2 Hoeper MM, Kramer T, Pan Z, et al. Mortality in pulmonary arterial hypertension: prediction by the 2015 European pulmonary hypertension guidelines risk stratification model. Eur Respir J 2017; 50: 1700740.

3 Kylhammar D, Kjellström B, Hjalmarsson C, et al. A comprehensive risk stratification at early follow-up determines prognosis in pulmonary arterial hypertension. Eur Heart J 2017; in press [https://doi.org/10.1093/eurheartj/ehx257].

4 Galiè N, Humbert M, Vachiery JL, et al. 2015 ESC/ERS Guidelines for the diagnosis and treatment of pulmonary hypertension: The Joint Task Force for the Diagnosis and Treatment of Pulmonary Hypertension of the European Society of Cardiology (ESC) and the European Respiratory Society (ERS). Eur Respir J 2015; 46: 903-975.

5 Galie N, Humbert M, Vachiery JL, et al. 2015 ESC/ERS Guidelines for the diagnosis and treatment of pulmonary hypertension: The Joint Task Force for the Diagnosis and Treatment of Pulmonary Hypertension of the European Society of Cardiology (ESC) and the European Respiratory Society (ERS). Eur Heart J 2016; 37: 67-119.

6 Harrell FE Jr, Lee KL, Califf RM, et al. Regression modelling strategies for improved prognostic prediction. Stat Med 1984; 3: 143-152. 